\title{
Re-mention of an old neurodegenerative disease: Alzheimer's disease
}

\author{
ZHANG Peng $^{1 *}$, ADAMS Ursula $^{2} \&$ YUAN ZengQiang ${ }^{1 *}$ \\ ${ }^{1}$ State Key Laboratory of Brain and Cognitive Sciences, Institute of Biophysics, Chinese Academy of Sciences, Beijing 100101, China; \\ ${ }^{2}$ Biological Sciences, The University of Chicago, Chicago, Illinois 60637, USA
}

Received August 24, 2012; accepted November 30, 2012

\begin{abstract}
Alzheimer's disease (AD), the most common form of neuropsychiatric disorder, is characterized by neuronal degeneration and inexorably progressing dementia, especially in the elderly population. With a rapidly aging population in both developed and developing countries, $\mathrm{AD}$ has emerged as one of the largest growing problems worldwide. Current drugs improve the symptoms of $\mathrm{AD}$, but do not have any profound intervention to delay its onset. Thus, understanding the molecular mechanisms underlying the genes tied to $\mathrm{AD}$ will be crucial to the development of therapeutic targets. This review will summarize the aetiology, pathology, and the evidence for the genetic components in $\mathrm{AD}$, discuss the proposed amyloid cascade and the following tau hyperphosphorylation hypothesises, oxidative stress mediated neuronal cell death, as well as the function of Retromer complex during the developing of AD. Our laboratory's current research progress and the challenges that still remained will be also highlighted.
\end{abstract}

Alzheimer's disease (AD), $\beta$-amyloid (A $\beta$ ), tau, oxidative stress, neuronal cell death, Retromer complex

Citation: $\quad$ Zhang P, Adams U, Yuan Z Q. Re-mention of an old neurodegenerative disease: Alzheimer's disease. Chin Sci Bull, 2013, 58: 1731-1736, doi: $10.1007 / \mathrm{s} 11434-013-5673-\mathrm{x}$

Alzheimer's disease (AD), the most common form of dementia, was first described by German neuropathologist Alois Alzheimer in 1906 [1]. In the autopsy report of a woman, who had died of a progressive cognitive disorder, Alois Alzheimer described two distinctive pathologies of the patient's brain: neurofibrillary tangles, and senile plaques, both of which have become synonymous with Alzheimer's disease [2].

With a rapidly aging population, in most societies dementia is becoming a major health burden. In 2009, 35.6 million cases of $\mathrm{AD}$ were reported worldwide, and the number could be more than doubled by 2050 [3]. Consequently, the total direct costs are estimated at $\$ 183$ billion, and are expected to increase to $\$ 1.1$ trillion by 2050 [4]. Because $\mathrm{AD}$ is degenerative and cannot be cured, most of the patients suffer an average of 4-8 years progression from the onset symptoms to death [5]. It places an exhausting and increasing burden on patients, caregivers and society.

*Corresponding authors (email: pengzhang@moon.ibp.ac.cn; zqyuan@ibp.ac.cn)

\section{Aetiology and pathology}

It has been about a century since the description of the disease was developed by Alzheimer; we still do not fully understand what causes Alzheimer's disease. Several lines of investigation generally indicate that there is unlikely to be a single clear "cause" of AD. It is more likely the result of a combination of inter-related factors, including aging, genetic factors, lifestyle factors, and environmental influences. Aging is the top risk factor for developing Alzheimer's disease. Most patients with $\mathrm{AD}$ are diagnosed at age 65 or older, these individuals are said to have "late-onset" AD. Nearly $10 \%$ of the population over the age of 65 , and $50 \%$ of the population over the age of 85 have Alzheimer's disease [6]. The probability of being diagnosed with AD nearly doubles every five years after age 65 . However, people younger than age 65 can also develop the disease, which is defined as "early-onset" AD. Two kinds of genes have been demonstrated as the genetic risk factors for $\mathrm{AD}$. The first is thought to be a "risk gene", ApoE 4 (the $\varepsilon 4$ allele of the 
apolipoprotein E, ApoE4), the mutant of which has been shown increased the likelihood of developing late-onset $\mathrm{AD}$ [7]. However, why the mutated gene or different isoforms increase the risk of disease is still unclear. By contrast, around $0.1 \%$ of the cases are autosomal dominant familial forms, which usually have an early-onset (before age 65) [8]. The early-onset autosomal dominant familial AD (FAD) is usually attributed to mutations in one of three genes: amyloid precursor protein (APP), Presenilin 1 (PSI; also known as PSEN1) and PS2 (also known as PSEN2) [9]. APP is a single-pass transmembrane protein which is expressed highly in the synapses of neurons, and it has been found as an important regulator of neural plasticity [10] and synapse formation [11]. APP processing generates the $\beta$-amyloid (A $\beta$ ) peptides, which are the main components of senile plaques in AD brains. Mutations in APP genes have been reported as the causal factors for early-onset FAD [12]. As the catalytic components of $\gamma$-secretase complex, PS1 and PS2 are involved in $A \beta$ production by the proteolytic processing of APP [13]. PS mutations have been presumed to cause FAD by increasing the ratio of the more toxic $A \beta 42$ isoform to A $\beta 40$ [14]. At present, more than 100 different mutations in PS1 and PS2 have been associated with early-onset FAD [15]. These arguments support a role of $A \beta$ in the pathogenesis of $\mathrm{AD}$, and result in the formulation of the "Amyloid cascade hypothesis", which will be further discussed in the next section.

Although age and genetic mutations are out of our control, researchers have identified several lifestyle factors that can reduce a person's risk of developing $\mathrm{AD}$, such as avoiding tobacco, limiting alcohol consumption, and engaging in intellectually stimulating activities [16]. Aluminum is one of the environmental factors that has been shown to be associated with both plaques and tangles in the $\mathrm{AD}$ brains [17], but no causal relationship has yet been proved. Formaldehyde, one of the most toxic organic compounds, has been found to induce tau to form amyloid-like aggregates [18]. The authors further demonstrated that the urine formaldehyde concentrations of the elderly Alzheimer's patients are significantly higher than that of normal elderly volunteers [19]. These data indicate that formaldehyde stress could be one of the mechanisms involved in the developing of $\mathrm{AD}$.

The neuropathologic changes of $\mathrm{AD}$ are characterized by loss of neurons and synapses in the neocortex and hippocampus regions [20]; both amyloid plaques and neurofibrillary tangles have been generally accepted as the hallmark lesions of AD. Senile plaques are mostly composed of insoluble $\mathrm{A} \beta$ deposits and extracellular materials around neurons. Neurofibrillary tangles are formed by the intracellular microtubule-associated protein tau which has become hyperphosphorylated [21]. Most studies agree that the classical pathological criteria for $\mathrm{AD}$ can account for $40 \%-70 \%$ of the cases [22], with additional pathologies such as Lewy body pathology [23] working together with AD pathology to account for an additional $20 \%-30 \%$ of dementia cases. Besides these hallmarks, over activation of the innate immune response and inflammatory processes are also observed [24]. However, whether the abnormalities in these pathological structures are the cause of the disease, or are only eventual reflections of the disease, remains elusive. So far, several lines of evidence have shown that there could be plausible cause-and-effect circuits that exist and exacerbate each other's detrimental effects [25].

\section{Amyloid cascade and tau hyperphosphoryla- tion-Two main hypotheses}

A lot of pathological and aetiological hypotheses have been proposed, including genetics, calcium homeostasis dysfunction, oxidative stress, vesicle trafficking disruption, and inflammation with the resultant axon transport dysfunctions and cognitive decline. However, $A \beta$ cascade combining with tau hyperphosphorylation is still the major regarded pathogenetic mechanism [26].

\subsection{Amyloid cascade hypothesis}

According to the "amyloid cascade hypothesis", $\mathrm{A} \beta$ deposition is the crucial pathological trigger in the disease. The accumulated $A \beta$ subsequently leads to the formation of NFTs and eventually neuron loss. Early work has indicated that the $A \beta$ neurotoxicity requires the peptides to be assembled into fibrillar, rather than soluble forms [27]. However, recent study demonstrates that soluble oligomeric $A \beta$ is the neurotoxic specie, but not the large amyloid deposit [28]. More studies demonstrate that $A \beta$ neurotoxicity depends on the interactions with copper ions, prion protein, and $\mathrm{N}$-methylD-aspartate receptors [29,30]. Both necrotic and apoptotic processes have been found in AD brains as well as in cultured neurons after exposed to $A \beta[31,32]$. However, the precise mechanisms by which $A \beta$ peptide triggers cell death remain largely unknown. It has been reported that $A \beta$ disturbs mitochondrial respiration and promotes the generation of reactive oxygen species (ROS) [33-35]. Furthermore, $\mathrm{A} \beta$-induced oxidative stress leads to neuronal cell death that can be inhibited by antioxidants [33,36,37]. Additionally, pathologic study has suggested that nitric oxide (NO) synthases (NOSs) also appeared to participate in the pathogenesis of AD [38], and PS1 mutation-induced neurotoxicity is inhibited by NOS inhibitors [39]. These findings suggest that NO and ROS may be important mediators of $A \beta$-induced neuronal cell death. However, how many downstream targets of ROS/NO in AD remain to be elucidated. A previous study demonstrated that $A \beta$-induced ROS activated the ASK1-JNK pathway, which plays an important role in the pathogenesis of $\mathrm{AD}$ [40]. Another way $\mathrm{A} \beta$ causes cell 
death is through increasing Bim levels while also down regulating Bcl-2 levels, leading to the activation of Bax and neuronal cell death [41]. In addition, up-regulation of AKT restored the $\mathrm{A} \beta$-induced alterations of the $\mathrm{Bcl}-2$ family members and suppressed the activation of JNK and caspase-3 [42]. Based on these evidences, selective interference targeting to $\mathrm{A} \beta$ generation and deposition could be utilized to treat or prevent AD.

\subsection{Tau hypothesis}

There are six tau isoforms existing in human brain, which are the products of alternative splicing from a single gene named MAPT (microtubule-associated protein tau) [43]. Tau proteins can stabilize tubulin and promote them assembly into microtubules [44]. Tau is a phosphoprotein that has 84 putative phosphorylation sites that could be modified by serine/threonine protein kinases [3]. These phosphorylation kinases can be divided in two types: (1) Proline-directed protein kinases (PDPKs), such as GSK3 $\beta$ [45], cdk5 [46], and kinases belonging to MAPKs family; (2) Non-prolinedirected protein kinase (NPDPK) such as protein kinase C (PKC), protein kinase A (PKA), partition defective1 (PAR1), and microtubule affinity regulating kinases (MARK) [47]. In neurodegeneration, tau is mainly modified by the prolinedirected protein kinase, GSK3 [47]. Hyperphosphorylated tau accumulates in the somatodendritic compartment of neurons, aggregates, and eventually forms NFTs [48]. It has been found that hyperphosphorylated tau causes mitochondrial dysfunction and induces oxidative stress [49]. Furthermore, the pathological tau disturbs axonal transport in $\mathrm{AD}$ neurons causing synapse starvation and ultimately neuronal damage [50]. In addition, recent studies have shown that phosphorylation of tau antagonizes apoptosis by stabilizing $\beta$-catenin $[51,52]$. The new findings reveal a dual role of tau phosphorylation in making the cells anti-apoptosis and leading to neurodegeneration. Although it is increasingly clear that tau may act as a common mediator of neurodegeneration for various upstream pathological events, a detailed map of causes and effects still remains elusive.

\subsection{Linking $A \beta$ and tau}

Based on the amyloid cascade hypothesis, $A \beta$ formation is the crucial step in the developing of AD. So, we ask where tau is to be placed in the amyloid cascade. Is tau a downstream target, or a paralleled effector of $\mathrm{A} \beta$ toxicity? Evidence from both in vivo and in vitro models suggests that there are three possible modes of interaction between these two pathologies: (1) A $\beta$ drives tau toxicity by causing hyperphosphorylation of tau; (2) A $\beta$ toxicity is critically dependent on the presence of tau; (3) A $\beta$ and tau are synergistic, thereby possibly amplifying each other's toxic effects [3].

\section{Research interests of our laboratory}

\subsection{Oxidative stress and neuronal cell death}

As discussed above, $A \beta$-induced oxidative stress leads to neuron apoptosis. However, the mechanisms underlying the biological effects of oxidative stress remain largely unknown. Mammalian Ste20-like kinases (MST) 1/2 and their Drosophila ortholog Hippo have been demonstrated that involved in diverse biological process ranging from organ size control, cell survival and death, to proliferation and tumorigenesis [53]. In our previous studies, we discovered that oxidative stress activates MST, activated MST1 phosphorylates Forkhead box O3 (FOXO3), and in turn, the active form of FOXO3 promotes neuronal cell death through induction of the proapoptotic gene BIM [54]. Furthermore, we and others found that MST activity was regulated by Akt-induced phosphorylation [55,56], and c-Jun N-terminal kinase (JNK) worked together with MST1 through a feedback manner during cell death [57]. Until then, the mechanisms that regulate MST1 in oxidative stress responses remained unclear. Subsequently, we demonstrated that oxidative stress induced the c-Abl-dependent tyrosine phosphorylation of MST1 and increased the interaction between MST1 and FOXO3, thereby activating the MST1-FOXO3 signalling pathway, leading to cell death in both primary culture neurons and rat hippocampal neurons [58]. Moreover, we recently discovered an evolutionarily conserved signalling link between c-Abl and MST2 in regulating the neuronal cell death that caused by Rotenone-induced oxidative stress [59].

Based on our previous findings, the roles of c-AblMST1/2-FOXO3 cascade in oxidative stress induced neuronal cell death have been revealed, and it will be important for us in future studies to determine the role of these pathways in the pathogenesis of AD by using gene manipulation in the rodent models.

\subsection{Retromer and APP processing}

The main function of Retromer, which has been well illustrated, is regulation of Wingless/Wnt secretion by recycling Wntless from early endosomes to the tans-Golgi network (TGN) [60,61]. Previous data indicate the Retromer to be functionally involved in the cellular trafficking of Memapsin 2 (BACE) and SorLA, both of which have been demonstrated playing important roles in regulating APP processing [62,63]. In addition, deficits in Retromer transport are associated with sporadic AD and pronounced neurodegeneration in AD animal models [64]. In 2008, Muhammad et al. [65] demonstrated that Retromer deficiency could contribute to disease pathogenesis observed in late-onset Alzheimer's disease using AD-mice and fly models. Another line of evidence endorsing that Retromer activity is associated with $\mathrm{AD}$ pathogenesis came from the clinical epide- 
miological studies. It has been reported that the mutations of SNX3 [61,66], which has been shown as an essential component of Retromer in sorting wntless, is genetically associated with $\mathrm{AD}$ [67]. These data supported an association between Retromer deficiency and neurodegeneration, and suggested parallel increases in $A \beta$ generation. A recent study demonstrated that the majority of APP is processed in the TGN to produce $A \beta 40$, with a requirement for the Retromer to sustain protein recycling from endosomes to the TGN [68]. However, the role of the Retromer in APP metabolism is still largely unknown. Retromer control of APP processing may be through either direct (retrieving APP from endosomes) or indirect (retrieving SorLA from endosomes and consequently retaining APP in the secretory pathway). In either case, disruption of Retromer is predicted to cause accumulation of APP or APP derivatives in late endosomal compartments [69].

Additionally, the cellular trafficking of BACE1 was also regulated by Retromer. Decreasing the expression of VPS26, a subunit of the Retromer complex, caused the accumulation of BACE1 in early endosomes [62]. Furthermore, Down-regulating the expression of SNX6, another putative subunit of Retromer, increases the intracellular levels of BACE1, and following the enhancement of sAPP $\beta$ and C99 levels [70]. Recently, the Retromer-associated sortilin has also been involved in the recycling of BACE1 from endosomes to TGN [71]. However, a wealth of study has suggested that the retrograde transport of APP was distinct from that of BACE1 [72]. Other studies also argue that BACE1 and APP are mainly located in different lipid rafts which can be fused together during endocytosis [73], and this precise regulation restricts the encounter of APP and BACE1 [74]. These studies suggest that further investigating the regulation roles of APP and BACE1 trafficking may offer new therapeutic targets for $\mathrm{AD}$ treatment, which has been developing extensively in our laboratory.

\section{Conclusions and future directions}

In summary, the exact etiopathogenesis of Alzheimer's disease is still obscure. The main neuropathology is the genesis and the defective clearance of the toxic fibrillar $A \beta$. The proposed mechanisms include the amyloid cascade hypothesis and the following tau hyperphosphorylation, vesicle trafficking disruption and oxidative stress. Current drugs target only the neurotransmitter dysfunction in $\mathrm{AD}$, and although they can improve the symptoms, they do not have profound disease-modifying effects [21]. Strategies or agents which modify the disrupted trafficking of APP/BACE1 reduce aggregation of $A \beta$, and therefore accelerate the clearance of neurotoxic amyloid may provide better cognitive advantage in AD.

Given that c-Abl-MST1/2 signalling transduction plays fundamental roles in oxidative stress induced neuronal cell death, the amount of upstream initiators or downstream sensors involved in this cascade still need further investigating. Additionally, the Retromer complex has been demonstrated as key regulator during AD development, although the mechanisms proposed by different researchers still remain controversial. Most of the current conclusions are generated by in vitro tissue cultural assays, and the biggest weakness of these assays is the difficulty in mimicking the real in vivo physiological/pathological processes. In this way, using the $\mathrm{AD}$ animal models (mutants or transgenic) to further investigate the underlying mechanisms should be a reasonable strategy. In addition, recent studies have shown that a missense mutation (D620N) of the VPS35 gene causes Parkinson's disease (PD) [75,76]. According to the previous crystal structure research, this mutation localizes near the interface between VPS35 and VPS29 [77]. Therefore, we speculate that this mutation causes PD potentially by disrupting the sorting efficiency of Retromer. Given that, it is also possible that this mutation exerts an effect on $\mathrm{AD}$ risk. Our laboratory is currently undergoing further investigation to test this hypothesis.

We apologize to authors whose work could not be cited directly owing to space limitations. We thank Yuan lab members for critical reading of the manuscript. This work was supported in part by the National Natural Science Foundation of China (81030025 and 81125010), and the National Basic Research Program of China (2009CB918704).

1 Alzheimer A. ber einen eigenartigen schweren erkrankungsprozess der hirnrinde. Neurologisches Centralblatt, 1906, 23: 1129-1136

2 Graeber M B, Kosel S, Egensperger R, et al. Rediscovery of the case described by Alois Alzheimer in 1911: Historical, histological and molecular genetic analysis. Neurogenetics, 1997, 1: 73-80

3 Ittner L M, Gotz J. Amyloid-beta and tau-A toxic pas de deux in Alzheimer's disease. Nat Rev Neurosci, 2011, 12: 65-72

4 Stefanacci R G. The costs of Alzheimer's disease and the value of effective therapies-page 2. Am J Manag Care, 2011, 17: S356-S362

5 Thies W, Bleiler L. 2011 Alzheimer's disease facts and figures. Alzheimers Dement, 2011, 7: 208-244

6 Zhang $\mathrm{Y} \mathrm{W}$, Thompson $\mathrm{R}$, Zhang $\mathrm{H}$, et al. App processing in Alzheimer's disease. Mol Brain, 2011, 4: 3

7 Corder E H, Saunders A M, Strittmatter W J, et al. Gene dose of apolipoprotein e type 4 allele and the risk of Alzheimer's disease in late onset families. Science, 1993, 261: 921-923

8 Blennow K, de Leon M J, Zetterberg H. Alzheimer's disease. Lancet, 2006, 368: 387-403

9 Waring S C, Rosenberg R N. Genome-wide association studies in Alzheimer disease. Arch Neurol, 2008, 65: 329-334

10 Turner P R, O'Connor K, Tate W P, et al. Roles of amyloid precursor protein and its fragments in regulating neural activity, plasticity and memory. Prog Neurobiol, 2003, 70: 1-32

11 Priller C, Bauer T, Mitteregger G, et al. Synapse formation and function is modulated by the amyloid precursor protein. J Neurosci, 2006, 26: 7212-7221

12 Zheng H, Koo E H. The amyloid precursor protein: Beyond amyloid. Mol Neurodegener, 2006, 1: 5

13 De Strooper B. Aph-1, pen-2, and nicastrin with presenilin generate an active gamma-secretase complex. Neuron, 2003, 38: 9-12

14 Hardy J, Selkoe D J. The amyloid hypothesis of Alzheimer's disease: Progress and problems on the road to therapeutics. Science, 2002, 297: 353-356 
15 Wolfe M S. When loss is gain: Reduced presenilin proteolytic function leads to increased abeta42/abeta40. Talking point on the role of presenilin mutations in Alzheimer disease. EMBO Rep, 2007, 8: $136-140$

16 Trojanowski J Q, Jedrziewski M K, Johnson B, et al. The art and science of anti-aging therapies. Sci Aging Knowledge Environ, 2005, 17: pe11

17 Crapper D R, Krishnan S S, Quittkat S. Aluminium, neurofibrillary degeneration and Alzheimer's disease. J Neurol, 1976, 99: 67-80

18 Nie C L, Wang X S, Liu Y, et al. Amyloid-like aggregates of neuronal tau induced by formaldehyde promote apoptosis of neuronal cells. BMC Neurosci, 2007, 8: 9

19 He R, Lu J, Miao J. Formaldehyde stress. Sci China Life Sci, 2010, 53: 1399-1404

20 Wenk G L. Neuropathologic changes in Alzheimer's disease. J Clin Psychiatry, 2003, 64 (Suppl 9): 7-10

21 Citron M. Alzheimer's disease: Strategies for disease modification. Nat Rev Drug Discov, 2010, 9: 387-398

22 O'Brien R J, Wong P C. Amyloid precursor protein processing and Alzheimer's disease. Annu Rev Neurosci, 2011, 34: 185-204

23 Riemenschneider M, Schoepfer-Wendels A, Friedrich P, et al. No association of vacuolar protein sorting 26 polymorphisms with Alzheimer's disease. Neurobiol Aging, 2007, 28: 883-884

24 McGeer P L, McGeer E G. Nsaids and Alzheimer disease: Epidemiological, animal model and clinical studies. Neurobiol Aging, 2007, 28: 639-647

25 Zhang B, Carroll J, Trojanowski J Q, et al. The microtubule-stabilizing agent, epothilone $\mathrm{d}$, reduces axonal dysfunction, neurotoxicity, cognitive deficits, and Alzheimer-like pathology in an interventional study with aged tau transgenic mice. J Neurosci, 2012, 32: 3601-3611

26 Mohandas E, Rajmohan V, Raghunath B. Neurobiology of Alzheimer's disease. Indian J Psychiatry, 2009, 51: 55-61

27 Tanzi R E, Bertram L. Twenty years of the Alzheimer's disease amyloid hypothesis: A genetic perspective. Cell, 2005, 120: 545-555

28 Walsh D M, Selkoe D J. A beta oligomers-A decade of discovery. J Neurochem, 2007, 101: 1172-1184

29 You H, Tsutsui S, Hameed S, et al. Abeta neurotoxicity depends on interactions between copper ions, prion protein, and $N$-methyl-Daspartate receptors. Proc Natl Acad Sci USA, 2012, 109: 1737-1742

30 Behbehani R G. A novel method for thermodynamic study on binding of copper ion with Alzheimer's amyliod $\beta$ peptide. Chin Sci Bull, 2009, 54: 1037-1042

31 Loo D T, Copani A, Pike C J, et al. Apoptosis is induced by betaamyloid in cultured central nervous system neurons. Proc Natl Acad Sci USA, 1993, 90: 7951-7955

32 Behl C, Davis J B, Klier F G, et al. Amyloid beta peptide induces necrosis rather than apoptosis. Brain Res, 1994, 645: 253-264

33 Behl C, Davis J B, Lesley R, et al. Hydrogen peroxide mediates amyloid beta protein toxicity. Cell, 1994, 77: 817-827

34 Hensley K, Carney J M, Mattson M P, et al. A model for betaamyloid aggregation and neurotoxicity based on free radical generation by the peptide: Relevance to Alzheimer disease. Proc Natl Acad Sci USA, 1994, 91: 3270-3274

35 Shearman M S, Ragan C I, Iversen L L. Inhibition of pc12 cell redox activity is a specific, early indicator of the mechanism of betaamyloid-mediated cell death. Proc Natl Acad Sci USA, 1994, 91: 1470-1474

36 Mattson M P, Goodman Y. Different amyloidogenic peptides share a similar mechanism of neurotoxicity involving reactive oxygen species and calcium. Brain Res, 1995, 676: 219-224

37 Pillot T, Drouet B, Queille S, et al. The nonfibrillar amyloid betapeptide induces apoptotic neuronal cell death: Involvement of its c-terminal fusogenic domain. J Neurochem, 1999, 73: 1626-1634

38 Vodovotz Y, Lucia M S, Flanders K C, et al. Inducible nitric oxide synthase in tangle-bearing neurons of patients with Alzheimer's disease. J Exp Med, 1996, 184: 1425-1433

39 Hashimoto Y, Ito Y, Arakawa E, et al. Neurotoxic mechanisms triggered by Alzheimer's disease-linked mutant m1461 presenilin 1: Involvement of no synthase via a novel pertussis toxin target. J
Neurochem, 2002, 80: 426-437

40 Kadowaki H, Nishitoh H, Urano F, et al. Amyloid beta induces neuronal cell death through ros-mediated ask1 activation. Cell Death Differ, 2005, 12: 19-24

41 Kudo W, Lee H P, Smith M A, et al. Inhibition of bax protects neuronal cells from oligomeric abeta neurotoxicity. Cell Death Dis, 2012, 3: e309

42 Yin G, Li L Y, Qu M, et al. Upregulation of akt attenuates amyloid-beta-induced cell apoptosis. J Alzheimers Dis, 2011, 25: 337-345

43 Goedert M, Spillantini M G, Jakes R, et al. Multiple isoforms of human microtubule-associated protein tau: Sequences and localization in neurofibrillary tangles of Alzheimer's disease. Neuron, 1989, 3: 519-526

44 Harada A, Oguchi K, Okabe S, et al. Altered microtubule organization in small-calibre axons of mice lacking tau protein. Nature, 1994, 369: 488-491

45 Ishiguro K, Shiratsuchi A, Sato S, et al. Glycogen synthase kinase 3 beta is identical to tau protein kinase $\mathrm{i}$ generating several epitopes of paired helical filaments. FEBS Lett, 1993, 325: 167-172

46 Ishiguro K, Kobayashi S, Omori A, et al. Identification of the $23 \mathrm{kDa}$ subunit of tau protein kinase ii as a putative activator of cdk5 in bovine brain. FEBS Lett, 1994, 342: 203-208

47 Avila J, Hernández F. Tau phosphorylation. In: Nixon R A, Yuan A, eds. Cytoskeleton of the Nervous System. New York: Springer, 2011. 73-82

48 Gotz J, Probst A, Spillantini M G, et al. Somatodendritic localization and hyperphosphorylation of tau protein in transgenic mice expressing the longest human brain tau isoform. EMBO J, 1995, 14: 1304-1313

49 David D C, Hauptmann S, Scherping I, et al. Proteomic and functional analyses reveal a mitochondrial dysfunction in p3011 tau transgenic mice. J Biol Chem, 2005, 280: 23802-23814

50 Reddy $\mathrm{P}$ H. Abnormal tau, mitochondrial dysfunction, impaired axonal transport of mitochondria, and synaptic deprivation in Alzheimer's disease. Brain Res, 2011, 1415: 136-148

51 Li H L, Wang H H, Liu S J, et al. Phosphorylation of tau antagonizes apoptosis by stabilizing beta-catenin, a mechanism involved in Alzheimer's neurodegeneration. Proc Natl Acad Sci USA, 2007, 104: 3591-3596

52 Wang J Z, Liu F. Microtubule-associated protein tau in development, degeneration and protection of neurons. Prog Neurobiol, 2008, 85: 148-175

53 Xiao L, Yuan Z. Redemystifying mst1/hippo signaling. Protein Cell, 2010, 1: 706-708

54 Lehtinen M K, Yuan Z, Boag P R, et al. A conserved mst-foxo signaling pathway mediates oxidative-stress responses and extends life span. Cell, 2006, 125: 987-1001

55 Jang S W, Yang S J, Srinivasan S, et al. Akt phosphorylates msti and prevents its proteolytic activation, blocking foxo3 phosphorylation and nuclear translocation. J Biol Chem, 2007, 282: 30836-30844

56 Yuan Z, Kim D, Shu S, et al. Phosphoinositide 3-kinase/akt inhibits mst1-mediated pro-apoptotic signaling through phosphorylation of threonine 120. J Biol Chem, 2010, 285: 3815-3824

57 Bi W, Xiao L, Jia Y, et al. C-jun n-terminal kinase enhances mst1mediated pro-apoptotic signaling through phosphorylation at serine 82. J Biol Chem, 2010, 285: 6259-6264

58 Xiao L, Chen $\mathrm{D}, \mathrm{Hu}$ P, et al. The c-abl-mst1 signaling pathway mediates oxidative stress-induced neuronal cell death. J Neurosci, 2011, 31: 9611-9619

59 Liu W, Wu J, Xiao L, et al. Regulation of neuronal cell death by c-abl-hippo/mst2 signaling pathway. PLoS One, 2012, 7: e36562

60 Belenkaya $\mathrm{T} \mathrm{Y}$, Wu Y, Tang X, et al. The retromer complex influences wnt secretion by recycling wntless from endosomes to the trans-golgi network. Dev Cell, 2008, 14: 120-131

61 Zhang P, Wu Y, Belenkaya T Y, et al. Snx3 controls wingless/wnt secretion through regulating retromer-dependent recycling of wntless. Cell Res, 2011, 21: 1677-1690

62 He X, Li F, Chang W P, et al. Gga proteins mediate the recycling pathway of memapsin 2 (bace). J Biol Chem, 2005, 280: 11696-11703 
63 Nielsen M S, Gustafsen C, Madsen P, et al. Sorting by the cytoplasmic domain of the amyloid precursor protein binding receptor sorla. Mol Cell Biol, 2007, 27: 6842-6851

64 Small SA, Kent K, Pierce A, et al. Model-guided microarray implicates the retromer complex in Alzheimer's disease. Ann Neurol, 2005, 58: 909-919

65 Muhammad A, Flores I, Zhang H, et al. Retromer deficiency observed in Alzheimer's disease causes hippocampal dysfunction, neurodegeneration, and abeta accumulation. Proc Natl Acad Sci USA, 2008, 105: 7327-7332

66 Harterink M, Port F, Lorenowicz M J, et al. A snx3-dependent retromer pathway mediates retrograde transport of the wnt sorting receptor wntless and is required for wnt secretion. Nat Cell Biol, 2011, 13: 914-923

67 Vardarajan B N, Bruesegem S Y, Harbour M E, et al. Identification of Alzheimer disease-associated variants in genes that regulate retromer function. Neurobiol Aging, 2012, 33: 2231. e15-2231. e30

68 Choy R W, Cheng Z, Schekman R. Amyloid precursor protein (app) traffics from the cell surface via endosomes for amyloid beta (abeta) production in the trans-golgi network. Proc Natl Acad Sci USA, 2012, 109: E2077-2082

69 Sullivan CP, Jay A G, Stack E C, et al. Retromer disruption promotes amyloidogenic app processing. Neurobiol Dis, 2011, 43: 338-345

70 Okada H, Zhang W, Peterhoff C, et al. Proteomic identification of sorting nexin 6 as a negative regulator of bace1-mediated app processing. FASEB J, 2010, 24: 2783-2794

71 Finan $\mathrm{G} \mathrm{M}$, Okada $\mathrm{H}$, Kim $\mathrm{T}$ W. Bacel retrograde trafficking is uniquely regulated by the cytoplasmic domain of sortilin. J Biol Chem, 2011, 286: 12602-12616

72 Ranganathan S, Noyes N C, Migliorini M, et al. Lrad3, a novel lowdensity lipoprotein receptor family member that modulates amyloid precursor protein trafficking. J Neurosci, 2011, 31: 10836-10846

73 Ehehalt R, Keller P, Haass C, et al. Amyloidogenic processing of the Alzheimer beta-amyloid precursor protein depends on lipid rafts. J Cell Biol, 2003, 160: 113-123

74 Tan J, Evin G. Beta-site app-cleaving enzyme 1 trafficking and Alzheimer's disease pathogenesis. J Neurochem, 2012, 120: 869-880

75 Vilarino-Guell C, Wider C, Ross O A, et al. Vps35 mutations in parkinson disease. Am J Hum Genet, 2011, 89: 162-167

76 Zimprich A, Benet-Pages A, Struhal W, et al. A mutation in vps35, encoding a subunit of the retromer complex, causes late-onset parkinson disease. Am J Hum Genet, 2011, 89: 168-175

77 Hierro A, Rojas A L, Rojas R, et al. Functional architecture of the retromer cargo-recognition complex. Nature, 2007, 449: 1063-1067

Open Access This article is distributed under the terms of the Creative Commons Attribution License which permits any use, distribution, and reproduction in any medium, provided the original author(s) and source are credited. 